\title{
Rendimiento por hectárea del maíz grano en México: distritos de riego vs temporal
}

\author{
Grain corn yield per hectare in Mexico: \\ irrigation districts vs temporal irrigation
}

\author{
José Luis Montesillo-Cedillo*
}

\section{Resumen}

La producción de maíz grano en México se lleva al cabo tanto en temporal como en sistemas de riego. Los sistemas de riego, sean unidades de riego para el desarrollo rural o distritos de riego propiamente dichos, se construyeron en las regiones áridas y semiáridas del país. Estas regiones abarcan alrededor de 60\% del territorio nacional. A los distritos de riego se les atribuye una superioridad numérica en términos de rendimiento, toneladas/hectárea, respecto del que se obtiene en temporal. El temporal propiamente dicho no requiere ningún sistema de riego. El objetivo del presente trabajo es comprobar si el rendimiento por hectárea de la producción de maíz grano en los distritos de riego es superior al obtenido en temporal. Para ello, se utiliza el análisis de varianza con datos referentes a dicha producción agrupados por distritos de riego al nivel nacional. Se consideran los años agrícolas desde 2001-2002 hasta el de 20132014. El año agrícola comprende del primero de octubre al último día de septiembre del año siguiente, y conjunta a los ciclos otoñoinvierno y primavera-verano. Se concluye que estadísticamente el rendimiento del maíz grano, ton/ha, producido en los distritos de riego es igual al de temporal con una probabilidad de $95 \%$, y que la producción en temporal tiene un menor costo social y ambiental, por lo menos en términos de agua.

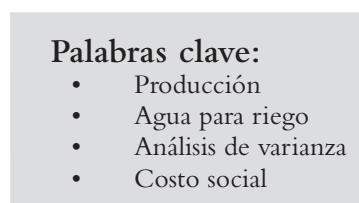

\begin{abstract}
The grain corn production in Mexico is made with natural water sources such as rain (temporal irrigation) and artificial irrigation systems. Irrigation systems for rural development or irrigation districts themselves, were built in the arid and semiarid regions around the country. These regions cover around $60 \%$ of it. A numerical superiority in terms of performance are attributed to irrigation districts, tons/hectare, compared to the amount obtained with temporal irrigation. The temporal irrigation does not requires irrigation system. The purpose of this work is to test out if the yield per hectare of grain corn production in irrigation districts is higher than the obtained with temporal irrigation. For this, the analysis of variance is used with data concerning to this production grouped per irrigation districts at national level. The agricultural years are considered from 2001-2002 to 2013-2014. An agricultural year comprehend from the first day of October to the last day of September of the following year, and this join the autumn-winter and spring-summer cycles. We conclude statistically that grain corn yield, tons/ha, produced in the irrigation districts is equal to the temporal irrigation with a probability of $95 \%$, and the temporal irrigation production has a lower social and environmental costs, at least in terms of water.
\end{abstract}

JEL: O13, Q14, R14

* Instituto de Estudios sobre la Universidad (IESU) de la Universidad Autónoma del Estado de México. jlmontesilloc@uaemex.mx 


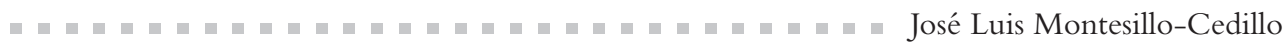

\section{Introducción}

En México, a partir de las primeras dos décadas del siglo xx y hasta la década de 1960 se construyeron los proyectos macro, encaminados principalmente a la construcción de grandes sistemas de riego -distritos de riego- los cuales permitieron ampliar la frontera agrícola y poblar las regiones desérticas y semidesérticas escasamente pobladas del país (Aguilar, 2010: 1).

Si bien se argumenta que las obras macro fueron para introducir el riego en las zonas de cultivo de temporal, es necesario matizar dichas afirmaciones, porque el riego se introdujo en las zonas áridas de cultivo (Vargas-Velazquez, 2010: 233) que por sus condiciones naturales, no son aptas para él, y las zonas aptas para el cultivo de temporal siguen siendo de temporal. Así, se tiene, de acuerdo con Mora (2003), que "las condiciones ecológicas de algunas zonas de México no son favorables para el desarrollo de actividades agrícolas, sin embargo, mediante mecanismos de riego principalmente en las zonas áridas y semiáridas se puede llevar a cabo el desarrollo e incremento de la actividad" [citado por Rivera, et al., (2012: 68)].

Además, cabe destacar que alrededor de $60 \%$ del territorio de México es árido (Cerutti, 2013: 3), y que 63\% de la superficie destinada al cultivo requiere del riego (Soto, 2003: 173). Por lo que "en México, las condiciones ecológicas imperantes no son favorables para la realización de las actividades agropecuarias, puesto que el relieve abrupto, la calidad de los suelos y las características del clima limitan considerablemente la disponibilidad natural de zonas aptas para el desarrollo de esta actividad" (Soto, 2003: 173). Los distritos de riego se abrieron en regiones áridas y semiáridas y precisan de grandes volúmenes de agua.

La actividad agrícola con sistemas de riego es la que más agua consume en México. La Comisión Nacional del Agua (Conagua, 2014a: 60) dice que el riego consume $61.82 \mathrm{~km}^{3}$ de los $81.65 \mathrm{~km}^{3}$ que se destinan a todos los usos consuntivos en México.

La utilización de $75.72 \%$ del total del agua destinada a los usos consuntivos por parte de la agricultura con sistemas de riego se justifica por su "alta productividad" respecto de la de temporal. "Cabe destacar que el rendimiento de la superficie bajo régimen de irrigación es superior al correspondiente a la agricultura de temporal. En 2013, para los principales cultivos por superficie cosechada -maíz grano, sorgo grano y frijol-, el rendimiento de los cultivos de riego, medido en ton/ha, fue de 2.2 a 3.3 veces mayor que el de los cultivos de temporal" (Conagua, 2014a: 89). 
En relación con el maíz grano, se afirma que "el rendimiento nacional alcanza en promedio las 3.2 ton/ha, siendo el rendimiento de temporal de 2.2 ton/ha y el de riego de 7.5 ton/ha" (sHcP, 2014: 1). De manera específica, se tiene que el rendimiento de maíz grano en el norte, producido con riego, es de 8 ton/ha, en tanto que el de temporal es de una; en el centro la relación es de 6.1 y de 3.7; en el sur es de 3.1 y de 2.8, respectivamente (Agroder, 2012: 4).

Las referencias anteriores, respecto del rendimiento por hectárea con riego, pasan por alto el hecho de que las zonas, regiones o estados en los que se abrieron los distritos de riego son áridas, por eso, "en general, puede observarse que la productividad, ton/ha, por regiones es muy baja, y serían comparables estos resultados a los obtenidos en varias zonas de buen temporal" (Soto, 2003: 185).

En estudios de caso se observan rendimiento en temporal similares al de los distritos de riego. Así, con base en el estudio de los efectos de la erosión del suelo en el rendiemiento de maíz en condiciones de temporal en Tomatal en Iguala, Gurrero, (Ocampo et al., 2007: 405) estimaron un rendimiento de entre 2.703 y 5.256 ton/ha. La variación del rendimiento está en función del nivel de erosión, pero el rendimiento máximo es similar al que se obtiene en los distritos de riego.

En el estudios de la fecha de siembra del frijol tenciopelo como cultivo de cobertera en el rendimiento de maíz (Castillo-Camaal, et al., 2011: 104-105) en condiciones de temporal sin riego de axulio, se concluye que el rendimiento va de las 2.9 hasta las 6.1 ton/ha. El rendimiento de 6.1 ton/ha es similar al de los distritos de riego.

El rendimiento del maíz por hectárea en el Valle Toluca-Atlacomulco va de las 4.2 hasta las 9.04 ton/ha, (Pérez, et al., 2007: 39). Cabe destacar que el máximo rendimiento es equivalente, y en la mayoría de los casos superior, al obtenido en los distritos de riego.

De lo expuesto líneas arriba se desprende el objetivo del presente trabajo: analizar el rendimiento, ton/ha, de maíz grano en los distritos de riego y en temporal durante los ciclos anuales de 2001-2002 hasta el de 2013-2014. Los datos utilizados están agrupados por distrito de riego, y se encuentra disponible en las "Estadísticas agrícolas de los distritos de riego" de la Conagua, la cual es sistematizada por el Instituto Mexicano de Tecnología del Agua (IMTA), disponible en <http://www.edistritos.com/DR/estadisticaAgricola/ distrito.php>.

El año agrícola comprende del primero de octubre al último día de septiembre del año siguiente, y conjunta los ciclos otoño-invierno y primaveraverano (Conagua, 2014b: 379). 
La relevancia de comprobar la superioridad del rendimiento por hectárea de maíz grano en los distritos de riego respecto de los de temporal es fundamental, toda vez que: "La problemática del agua de riego y en general del agua es, no sólo un problema técnico a resolver, es también un problema social, de cultura y del poder que detentan los distintos actores en la apropiación y gestión del agua" (Palmer, et al., s.f: 5). En consecuencia, de existir dicha superioridad habrá, naturalmente, más apoyos gubernamentales y hasta sociales a los productores de los distritos de riego y se podrían justificar las ingentes cantidades de agua que consumen, $25.61 \mathrm{~km}^{3}$ por año (Conagua, 2014a: 90).

Sin embargo, si la afirmación de que el rendimiento por hectárea de riego es superior al de temporal no se puede soportar con la evidencia, esto debe tener consecuencias en las políticas hidroagrícolas y la toma de decisiones (Conagua, 2014b: 1). Si dicha aseveración es verdadera no hay consecuencias excepto los resultados obtenidos actualmente, pero si no es cierta, sí hay consecuencias y resultados no previstos ni esperados, y probablemente no deseados en detrimento de los productores de maíz grano en temporal; que dicho sea de paso, son quienes conservan a las más de 59 variedades de maíz nativas de México, las cuales son la base de los más de 600 preparados comestibles de la cocina pluricultural de los mexicanos. En tanto que la producción de los distritos de riego se concentra en el maíz amarillo y en el maíz blanco ( $\mathrm{Tu}-$ rrent, et al.: 9).

Es clara la relevancia de conocer el rendimiento por hectárea de maíz grano en los distritos de riego y en temporal, y comprobar si el de riego es hasta tre veces superior al de temporal. Los distritos de riego consumen agua y reciben transferencias federales y estales, al amparo de su "mayor productividad" y de "producir" los alimentos que la población necesita (Sagarpa, 2008-2012: 9; Conagua, 2014: 3).

\section{Algunas precisiones teóricas}

Antes de realizar el análisis indicado es necesario aclarar que la comparación se realiza desde el punto de vista estadístico, lo cual no implica que desde el punto de vista teórico dicha comparación sea válida. Desde el punto de vista teórico las comparaciones se hacen entre iguales, por decirlo de una manera coloquial, pero comprensible por todos, y aunque se compare la producción de maíz grano, los procesos productivos no son comparables, toda vez que no se utilizan las mismas cantidades de factores, el proceso productivo de los distritos de riego utiliza un factor que los de temporal no: agua. 
En términos formales, el conjunto de cantidades necesarias de factores $[V(\gamma)]$ para la producción de maíz grano con riego puede representarse como: y la de temporal como: En donde, de acuerdo con Varian (1992: 4-6), $x$ es un vector de factores que pueden producir $y$ unidades, y ambos están contenidos en el conjunto de posibilidades de producción $(Y)$. De aquí se desprende que si $x \neq x^{1}$, tal que $x>x^{1}$, se espere $y>y^{1}$.

El solo hecho de que $x>x^{1}$ implica la no comparabilidad de ambos procesos de producción, y obliga a que la producción de maíz grano con agua para riego sea superior a la de temporal, lo cual no implica bajo ninguna circunstancia o modalidad que la producción con riego sea superior a la de temporal, porque son procesos no comparables, como ya se señaló.

En suma, decir que la producción con agua para riego es superior a la de temporal no tiene ningún fundamento teórico como ya quedó asentado líneas arriba con base en $[V(\gamma)]$. Pero sí tiene consecuencias de política económica, porque con ello se justifica la transferencia de recursos federales -como el agua, entre otros- a los estados o regiones cuyos procesos productivos tienen un factor de más respecto de los de temporal en detrimento de estos últimos.

\section{Principios básicos del análisis de varianza}

La comparación del rendimiento (toneladas por hectárea) de maíz grano entre los distritos de riego y de temporal se hace con base en el análisis de varianza, el cual permite comparar dos o más valores medios. En este caso, la comparación del rendimiento por hectárea se hace con base en los valores medios nacional para cada uno de los años agrícolas desde el de 2001-2002 hasta el de 2013-2014.

El análisis de varianza requiere del cumplimiento de los siguientes principios teóricos para que los resultados obtenidos tengan sentido estadístico: i) las muestras son independientes y aleatorias; ii) las muestras deben provenir de poblaciones normales y, iii) las poblaciones deben tener varianzas iguales.

Con la finalidad de garantizar lo adecuado del análisis de varianza realizado en el presente trabajo, primero se comprueba si las muestras cumplen con los requisitos teóricos. En este sentido, respecto de la independencia podemos decir que sí lo cumplen, puesto que una muestra es de los distritos de riego y la otra de temporal. En relación con la aleatoriedad también lo cumplen, porque el resultado observado (Wackerly, et al., 2002: 629) en el año agrícola $z$ de producción es uno de un conjunto infinito 
En relación con el principio de normalidad, debemos tener presente que si las distribuciones a partir de las cuales se obtienen las muestras no son altamente asimétricas, no será estrictamente necesario apegarse al principio de normalidad (Stevenson, 2002: 322). A lo cual nos apegamos, toda vez que el análisis de varianza es "bastante robusto con respecto a la suposición de normalidad (incluso desviaciones moderadas de esta suposición no cambia mucho los resultados)" (Kohler, 1999: 484). Los resultados de las pruebas de normalidad se pueden observar en el cuadro 1.

\section{Cuadro I}

Prueba de normalidad y grado de asimetría por año agrícola de las muestras del rendimiento de maíz grano, promedio nacional, ton/ha, de temporal y de los distritos de riego

\begin{tabular}{|c|c|c|c|c|c|}
\hline \multirow{2}{*}{$\begin{array}{c}\text { Año } \\
\text { agrícola }\end{array}$} & \multicolumn{7}{|c|}{ Temporal } \\
\cline { 2 - 6 } & Asimetría & Curtosis & J-B & Probabilidad de normalidad & Observaciones \\
\hline $2001-2002$ & 1.49 & 4.46 & 8.34 & 0.01 & 18 \\
\hline $2002-2003$ & 0.84 & 3.05 & 2.34 & 0.31 & 20 \\
\hline $2003-2004$ & 0.73 & 2.88 & 1.46 & 0.48 & 16 \\
\hline $2004-2005$ & 0.37 & 2.30 & 0.70 & 0.70 & 16 \\
\hline $2005-2006$ & 0.24 & 1.92 & 0.87 & 0.65 & 14 \\
\hline $2006-2007$ & 0.67 & 2.16 & 1.46 & 0.48 & 12 \\
\hline $2007-2008$ & 0.66 & 2.67 & 0.93 & 0.63 & 11 \\
\hline $2008-2009$ & 0.33 & 1.85 & 0.81 & 0.67 & 10 \\
\hline $2009-2010$ & 1.26 & 4.38 & 4.11 & 0.13 & 13 \\
\hline $2010-2011$ & 0.47 & 2.14 & 0.68 & 0.71 & 11 \\
\hline $2011-2012$ & 0.01 & 1.41 & 1.37 & 0.50 & 10 \\
\hline $2012-2013$ & -0.02 & 1.95 & 0.51 & 0.77 & 0.83 \\
\hline $2013-2014$ & -0.25 & 2.20 & 0.37 & & 12 \\
\hline
\end{tabular}


Economía Informa núm. 398 mayo - junio • 2016

\begin{tabular}{|c|c|c|c|c|c|}
\hline \multirow{2}{*}{$\begin{array}{c}\text { Año } \\
\text { agrícola }\end{array}$} & \multicolumn{7}{|c|}{ Riego } \\
\hline & Asimetría & Curtosis & J-B & Probabilidad de normalidad & Observaciones \\
\hline $2001-2002$ & 0.47 & 2.32 & 4.30 & 0.12 & 76 \\
\hline $2002-2003$ & 0.58 & 2.54 & 4.91 & 0.08 & 75 \\
\hline $2003-2004$ & 0.46 & 2.32 & 4.13 & 0.13 & 75 \\
\hline $2004-2005$ & 0.44 & 2.33 & 3.89 & 0.14 & 77 \\
\hline $2005-2006$ & 0.42 & 2.12 & 4.56 & 0.10 & 74 \\
\hline $2006-2007$ & 0.37 & 2.28 & 3.24 & 0.20 & 72 \\
\hline $2007-2008$ & 0.33 & 2.38 & 2.48 & 0.29 & 72 \\
\hline $2008-2009$ & 0.41 & 2.27 & 3.64 & 0.16 & 68 \\
\hline $2009-2010$ & 0.69 & 2.47 & 6.23 & 0.04 & 67 \\
\hline $2010-2011$ & 0.82 & 3.07 & 7.35 & 0.02 & 67 \\
\hline $2011-2012$ & 0.37 & 2.16 & 3.48 & 0.17 & 66 \\
\hline $2012-2013$ & 0.54 & 2.57 & 3.82 & 0.15 & 0.30 \\
\hline $2013-2014$ & 0.27 & 2.24 & 2.40 & & 73 \\
\hline
\end{tabular}

Nota: tenga presente que para una variable normalmente distribuida los valores de asimetría y curtosis son 0 y 3 , respectivamente; J-B se refiere a la prueba de normalidad de Jarque-Bera, y cuando la muestra es normal, J-B tiende a 1, no olvidemos que la prueba J-B se desarrolló para muestras grandes, desde el punto de vista estadístico.

Fuente: elaboración propia con base en información del IMTA (Instituto Mexicano de Tecnología del Agua) (2015), < http://www.edistritos.com/DR/estadisticaAgricola/distrito.php> el 05 de marzo de 2015.

Los resultados expuestos en el cuadro 1 permiten decir que estadísticamente las muestras de temporal provienen de poblaciones normalmente distribuidas, excepto la del ciclo 2001-2002. Por su parte, las muestras de los distritos de riego no son altamente asimétricas, como lo exige el análisis de varianza, toda vez que, de acuerdo con la prueba de normalidad, su probabilidad de provenir de una población normal es baja, no está de más recordar que esto depende del tamaño de la muestra considerada.

La prueba de igualdad de varianza de las muestras se presenta en el cuadro 2. Para dicha prueba se utilizaron las varianzas de las muestras y se aplicó la prueba F. Se recurrío al convencionalismo de colocar la varianza mayor en el numerador. El requisito de igualdad de varianza, a diferencia del de normalidad, sí debe cumplirse, porque su incumplimiento sí afecta seriamente la validez de la prueba (Kohler, 1999: 484).

Con base en los datos del cuadro 2 se puede decir que las varianzas de las muestras del rendimiento (ton/ha) medio de maíz grano de temporal y de los distritos de riego, sí satisfacen el principio teórico de igualdad de las varianzas. Toda vez que dichas varianzas son iguales desde el punto de vista estadístico a 5\% de significancia, con excepción de los años agrícolas 2004-2005 y 20072008 que no son significativos a dicho nivel, sino a 1 por ciento. 


\section{Cuadro 2}

\section{Prueba de igualdad de las varianzas de las muestras del rendimiento de maíz grano en toneladas por hectárea de los distritos de riego y de temporal}

\begin{tabular}{|c|c|c|c|c|}
\hline $\begin{array}{c}\text { Año } \\
\text { agrícola }\end{array}$ & $\begin{array}{c}\text { Varianza } \\
\text { de riego }\end{array}$ & $\begin{array}{c}\text { Varianza } \\
\text { de temporal }\end{array}$ & F calculado & $\begin{array}{c}\text { F de tablas al 5\% de } \\
\text { significancia }\end{array}$ \\
\hline $2001-2002$ & 4.16 & 4.16 & $(4.16 / 4.16)=1.00$ & $\mathrm{~F}_{(17,75)}=1.79$ \\
\hline $2002-2003$ & 4.20 & 3.97 & $(4.20 / 3.97)=1.06$ & $\mathrm{~F}_{(74,19)}=1.96$ \\
\hline $2003-2004$ & 4.24 & 5.78 & $(5.78 / 4.24)=1.36$ & $\mathrm{~F}_{(15,74)}=1.79$ \\
\hline $2004-2005$ & 4.56 & 2.04 & $(4.56 / 2.04)=2.24$ & $\mathrm{~F}_{(76,15)}=2.14$ \\
\hline $2005-2006$ & 4.84 & 2.98 & $(4.84 / 2.98)=1.62$ & $\mathrm{~F}_{(73,14)}=2.21$ \\
\hline $2006-2007$ & 4.88 & 2.59 & $(4.88 / 2.59)=1.88$ & $\mathrm{~F}_{(72,13)}=2.28$ \\
\hline $2007-2008$ & 5.73 & 2.25 & $(5.73 / 2.25)=2.55$ & $\mathrm{~F}_{(70,11)}=2.49$ \\
\hline $2008-2009$ & 5.77 & 3.28 & $(5.77 / 3.28)=1.76$ & $\mathrm{~F}_{(71,10)}=2.61$ \\
\hline $2009-2010$ & 5.81 & 5.88 & $(5.88 / 5.81)=1.01$ & $\mathrm{~F}_{(11,67)}=1.92$ \\
\hline $2010-2011$ & 5.51 & 4.09 & $(5.51 / 4.09)=1.35$ & $\mathrm{~F}_{(65,9)}=2.77$ \\
\hline $2011-2012$ & 6.85 & 3.26 & $(6.85 / 3.26)=2.10$ & $\mathrm{~F}_{(66,12)}=2.37$ \\
\hline $2012-2013$ & 6.30 & 2.55 & $(6.30 / 2.55)=2.47$ & $\mathrm{~F}_{(66,10)}=2.60$ \\
\hline $2013-2014$ & 7.36 & 3.60 & $(7.36 / 3.60)=2.04$ & $\mathrm{~F}_{(65,9)}=2.77$ \\
\hline
\end{tabular}

Nota: * denota significancias al 1\%, pero no al 5\%. Los valores $\mathrm{F}$ de tablas se tomaron de Kohter (1999: 923-926)

Fuente: elaboración propia con base en los datos del ImTA (Instituto Mexicano de Tecnología del Agua) (2015), < http://www.edistritos.com/DR/estadisticaAgricola/distrito.php > el 05 de marzo de 2015.

De las pruebas estadísticas realizadas referentes a la independencia y aleatoriedad, a la normalidad de la población de donde se extrajeron las muestras y a la igualdad de las varianzas poblacionales, se puede concluir que sí es factible realizar el análisis de varianza del rendimiento medio al nivel nacional por hectárea de maíz grano de los distritos de riego y de temporal.

La hipótesis nula $\left(\mathrm{H}_{0}\right)$ es: los rendimientos medios son iguales.

La hipótesis alterna $\left(\mathrm{H}_{1}\right)$ es: el rendimiento medio de los distritos de riego es superior al de temporal.

$\mathrm{Si}_{0}$ se rechaza, se concluye que probablemente las muestras no provengan de poblaciones que tengan medias iguales y, por lo tanto, el rendimiento de maíz grano de los distritos de riego sí sea superior al de temporal. En el caso contrario, se concluye que probablemente las muestras provengan de 
poblaciones con medias iguales, y que no hay superioridad alguna en el rendimiento de maíz grano producido en los distritos de riego respecto del de temporal.

\section{Análisis de varianza de la producción de maíz grano de los distritos de riego y de temporal}

El análisis de varianza permite probar la igualdad de los valores medios de las muestras, en este caso, se prueba si la media del rendimiento de maíz grano por hectárea de los distritos de riego es igual a la de temporal $\left(\mathrm{H}_{0}\right)$, esto es, las dos muestra fueron extraídas de la misma población o sus medias son iguales. El análisis se realiza por año agrícola. Cabe destacar que el tamaño de las muestras de los distritos de riego en todos los años son mayores a las de temporal (véase cuadro 1), y en todos los casos el número de muestras independientes es de dos (una de los distritos de riego y otra de temporal).

A partir del cuadro 3 y hasta el 15 se presentan los resultados obtenidos del análisis de varianza para cada uno de los años agrícolas.

\section{Cuadro 3}

Análisis de varianza para el año agrícola 2001-2002

\begin{tabular}{|l|r|r|r|r|c|}
\hline Fuente de variación & $\begin{array}{c}\text { Suma de } \\
\text { cuadrados }\end{array}$ & $\begin{array}{c}\text { Grados de } \\
\text { libertad (g.1) }\end{array}$ & $\begin{array}{c}\text { Varianza } \\
\text { estimada }\end{array}$ & $\begin{array}{c}\text { F } \\
\text { calculada }\end{array}$ & $\begin{array}{c}\text { F de tablas al 5\% } \\
\text { de significancia }\end{array}$ \\
\hline Medias intermediantes & 106.54 & $2-1=1$ & 106.54 & \\
\hline Medias internas & 24429.00 & $18+76-2=92$ & 249.27 & 0.401 & 3.94 \\
\hline Total & 24535.54 & 93 & 263.82 & & \\
\hline
\end{tabular}

Fuente: elaboración propia.

\section{Cuadro 4}

\section{Análisis de varianza para el año agrícola 2002-2003}

\begin{tabular}{|l|r|r|r|r|c|}
\hline Fuente de variación & $\begin{array}{c}\text { Suma de } \\
\text { cuadrados }\end{array}$ & $\begin{array}{c}\text { Grados de } \\
\text { libertad (g.1) }\end{array}$ & $\begin{array}{c}\text { Varianza } \\
\text { estimada }\end{array}$ & $\begin{array}{c}\text { F } \\
\text { calculada }\end{array}$ & $\begin{array}{c}\text { F de tablas al 5\% } \\
\text { de significancia }\end{array}$ \\
\hline Medias intermediantes & 49.96 & $2-1=1$ & 49.96 & \\
\hline Medias internas & 24846.17 & $20+75-2=93$ & 267.16 & 0.187 & 3.94 \\
\hline Total & 24896.13 & 94 & 264.85 & & \\
\hline
\end{tabular}

Fuente: elaboración propia. 


\section{Cuadro 5}

Análisis de varianza para el año agrícola 2003-2004

\begin{tabular}{|l|r|r|r|r|r|}
\hline Fuente de variación & $\begin{array}{c}\text { Suma de } \\
\text { cuadrados }\end{array}$ & $\begin{array}{c}\text { Grados de } \\
\text { libertad (g.1) }\end{array}$ & $\begin{array}{r}\text { Varianza } \\
\text { estimada }\end{array}$ & $\begin{array}{c}\text { F } \\
\text { calculada }\end{array}$ & $\begin{array}{c}\text { F de tablas al 5\% } \\
\text { de significancia }\end{array}$ \\
\hline Medias intermediantes & 33.70 & $2-1=1$ & 33.70 & \\
\hline Medias internas & 24932.16 & $16+75-2=89$ & 280.14 & 0.120 & 3.96 \\
\hline Total & 24965.86 & 90 & 277.398 & & \\
\hline
\end{tabular}

Fuente: elaboración propia.

\section{Cuadro 6}

Análisis de varianza para el año agrícola 2004-2005

\begin{tabular}{|l|r|r|r|r|r|}
\hline Fuente de variación & $\begin{array}{c}\text { Suma de } \\
\text { cuadrados }\end{array}$ & $\begin{array}{c}\text { Grados de } \\
\text { libertad (g.l) }\end{array}$ & $\begin{array}{r}\text { Varianza } \\
\text { estimada }\end{array}$ & $\begin{array}{c}\text { F } \\
\text { calculada }\end{array}$ & $\begin{array}{c}\text { F de tablas al 5\% } \\
\text { de significancia }\end{array}$ \\
\hline Medias intermediantes & 118.72 & $2-1=1$ & 118.72 & \\
\hline Medias internas & 27323.71 & $16+77-2=91$ & 300.26 & 0.395 & 3.94 \\
\hline Total & 27442.43 & 92 & 298.29 & & \\
\hline
\end{tabular}

Fuente: elaboración propia.

\section{Cuadro 7}

Análisis de varianza para el año agrícola 2005-2006

\begin{tabular}{|l|r|r|r|r|r|}
\hline Fuente de variación & $\begin{array}{c}\text { Suma de } \\
\text { cuadrados }\end{array}$ & $\begin{array}{c}\text { Grados de } \\
\text { libertad (g.1) }\end{array}$ & $\begin{array}{r}\text { Varianza } \\
\text { estimada }\end{array}$ & $\begin{array}{c}\text { F } \\
\text { calculada }\end{array}$ & $\begin{array}{c}\text { F de tablas al 5\% } \\
\text { de significancia }\end{array}$ \\
\hline Medias intermediantes & 20.60 & $2-1=1$ & 20.60 & \\
\hline Medias internas & 26781.58 & $15+74-2=87$ & 307.83 & 0.067 & 3.94 \\
\hline Total & 26802.18 & 88 & 304.57 & \\
\hline
\end{tabular}

Fuente: elaboración propia

\section{Cuadro 8}

Análisis de varianza para el año agrícola 2006-2007

\begin{tabular}{|l|r|r|r|r|r|}
\hline Fuente de variación & $\begin{array}{c}\text { Suma de } \\
\text { cuadrados }\end{array}$ & $\begin{array}{r}\text { Grados de } \\
\text { libertad }(\mathrm{g} .1)\end{array}$ & $\begin{array}{r}\text { Varianza } \\
\text { estimada }\end{array}$ & $\begin{array}{c}\text { F } \\
\text { calculada }\end{array}$ & $\begin{array}{c}\text { F de tablas al 5\% } \\
\text { de significancia }\end{array}$ \\
\hline Medias intermediantes & 71.74 & $2-1=1$ & 71.74 & \\
\hline Medias internas & 26111.15 & $14+73-2=85$ & 307.19 & 0.233 & 3.94 \\
\hline Total & 26182.89 & 86 & 304.45 & & \\
\hline
\end{tabular}

Fuente: elaboración propia. 


\section{Cuadro 9}

Análisis de varianza para el año agrícola 2007-2008

\begin{tabular}{|l|r|r|r|r|r|}
\hline Fuente de variación & $\begin{array}{c}\text { Suma de } \\
\text { cuadrados }\end{array}$ & $\begin{array}{c}\text { Grados de } \\
\text { libertad (g.1) }\end{array}$ & $\begin{array}{r}\text { Varianza } \\
\text { estimada }\end{array}$ & $\begin{array}{c}\text { F } \\
\text { calculada }\end{array}$ & $\begin{array}{c}\text { F de tablas al 5\% } \\
\text { de significancia }\end{array}$ \\
\hline Medias intermediantes & 96.02 & $2-1=1$ & 96.02 & \\
Medias internas & 29573.23 & $12+72-2=82$ & 360.65 & 0.266 & 3.94 \\
\hline Total & 29669.25 & 83 & 357.46 & & \\
\hline
\end{tabular}

Fuente: elaboración propia.

\section{Cuadro 10}

Análisis de varianza para el año agrícola 2008-2009

\begin{tabular}{|l|r|r|r|r|r|}
\hline Fuente de variación & $\begin{array}{c}\text { Suma de } \\
\text { cuadrados }\end{array}$ & $\begin{array}{c}\text { Grados de } \\
\text { libertad (g.1) }\end{array}$ & $\begin{array}{r}\text { Varianza } \\
\text { estimada }\end{array}$ & $\begin{array}{c}\text { F } \\
\text { calculada }\end{array}$ & $\begin{array}{c}\text { F de tablas al 5\% } \\
\text { de significancia }\end{array}$ \\
\hline Medias intermediantes & 88.86 & $2-1=1$ & 88.86 & \\
\hline Medias internas & 29841.83 & $11+72-2=81$ & 368.42 & 0.241 & 3.96 \\
\hline Total & 29930.68 & 82 & 365.01 & & \\
\hline
\end{tabular}

Fuente: elaboración propia.

\section{Cuadro II}

Análisis de varianza para el año agrícola 2009-2010

\begin{tabular}{|l|r|r|r|r|r|}
\hline Fuente de variación & $\begin{array}{c}\text { Suma de } \\
\text { cuadrados }\end{array}$ & $\begin{array}{c}\text { Grados de } \\
\text { libertad (g.1) }\end{array}$ & $\begin{array}{r}\text { Varianza } \\
\text { estimada }\end{array}$ & $\begin{array}{c}\text { F } \\
\text { calculada }\end{array}$ & $\begin{array}{c}\text { F de tablas al 5\% } \\
\text { de significancia }\end{array}$ \\
\hline Medias intermediantes & 37.76 & $2-1=1$ & 37.76 & \\
\hline Medias internas & 27579.63 & $12+68-2=78$ & 353.58 & 0.107 & 3.96 \\
\hline Total & 27617.39 & 79 & 349.59 & \\
\hline
\end{tabular}

Fuente: elaboración propia.

\section{Cuadro 12}

Análisis de varianza para el año agrícola 2010-2011

\begin{tabular}{|l|r|r|r|r|r|}
\hline Fuente de variación & $\begin{array}{c}\text { Suma de } \\
\text { cuadrados }\end{array}$ & $\begin{array}{c}\text { Grados de } \\
\text { libertad (g.1) }\end{array}$ & $\begin{array}{r}\text { Varianza } \\
\text { estimada }\end{array}$ & $\begin{array}{c}\text { F } \\
\text { calculada }\end{array}$ & $\begin{array}{c}\text { F de tablas al 5\% } \\
\text { de significancia }\end{array}$ \\
\hline Medias intermediantes & 31.66 & $2-1=1$ & 31.66 & \\
\hline Medias internas & 23990.92 & $10+66-2=74$ & 324.20 & 0.098 & 3.96 \\
\hline Total & 24022.59 & 75 & 320.30 & \\
\hline
\end{tabular}

Fuente: elaboración propia. 


\section{Cuadro I3}

Análisis de varianza para el año agrícola 2011-2012

\begin{tabular}{|l|c|r|r|r|c|} 
Fuente de variación & $\begin{array}{c}\text { Suma de } \\
\text { cuadrados }\end{array}$ & $\begin{array}{c}\text { Grados de } \\
\text { libertad }(\text { g.1) }\end{array}$ & $\begin{array}{r}\text { Varianza } \\
\text { estimada }\end{array}$ & $\begin{array}{c}\text { F } \\
\text { calculada }\end{array}$ & $\begin{array}{c}\text { F de tablas al 5\% } \\
\text { de significancia }\end{array}$ \\
\hline Medias intermediantes & 105.56 & $2-1=1$ & 105.56 & \\
\hline Medias internas & 30825.88 & $13+67-2=78$ & 395.20 & 0.267 & 3.96 \\
\hline Total & 30931.44 & 79 & 391.54 & & \\
\hline
\end{tabular}

Fuente: elaboración propia.

\section{Cuadro 14}

Análisis de varianza para el año agrícola 2012-2013

\begin{tabular}{|l|r|r|r|r|r|}
\hline Fuente de variación & $\begin{array}{c}\text { Suma de } \\
\text { cuadrados }\end{array}$ & $\begin{array}{c}\text { Grados de } \\
\text { libertad (g.1) }\end{array}$ & $\begin{array}{r}\text { Varianza } \\
\text { estimada }\end{array}$ & $\begin{array}{c}\text { F } \\
\text { calculada }\end{array}$ & $\begin{array}{c}\text { F de tablas al 5\% } \\
\text { de significancia }\end{array}$ \\
\hline Medias intermediantes & 42.03 & $2-1=1$ & 42.03 & \\
\hline Medias internas & 28159.70 & $11+67-2=76$ & 370.52 & 0.113 & 3.96 \\
\hline Total & 28201.73 & 77 & 366.26 & & \\
\hline
\end{tabular}

Fuente: elaboración propia.

\section{Cuadro I5}

Análisis de varianza para el año agrícola 2013-2014

\begin{tabular}{|l|r|r|r|r|r|}
\hline Fuente de variación & $\begin{array}{c}\text { Suma de } \\
\text { cuadrados }\end{array}$ & $\begin{array}{c}\text { Grados de } \\
\text { libertad (g.l) }\end{array}$ & $\begin{array}{r}\text { Varianza } \\
\text { estimada }\end{array}$ & $\begin{array}{c}\text { F } \\
\text { calculada }\end{array}$ & $\begin{array}{c}\text { F de tablas al 5\% } \\
\text { de significancia }\end{array}$ \\
\hline Medias intermediantes & 64.44 & $2-1=1$ & 64.44 & \\
\hline Medias internas & 31891.60 & $10+66-2=74$ & 430.97 & 0.149 & 3.96 \\
\hline Total & 31956.04 & 75 & 426.08 & & \\
\hline
\end{tabular}

Fuente: elaboración propia.

Con base en los resultados del análisis de varianza mostrados en los cuadros desde el tres hasta el 15 para cada uno de los años agrícolas referentes al rendimiento por hectárea de maíz grano, permiten no rechazar la hipótesis nula: estadísticamente la media del rendimiento de maíz grano en toneladas por hectárea de temporal es igual a la de los distritos de riego. Si bien es claro que numéricamente el rendimiento de los distritos de riego es superior al de temporal, estadísticamente el rendimiento es el mismo a 95\% de probabilidad. En suma, los valores medios de las poblaciones de las que se extrajeron las muestran son iguales. 


\section{Conclusiones}

Con base en el análisis de varianza se puede concluir que el rendimiento de maíz grano producido en los distritos de riego es igual al de temporal, por lo tanto, no hay evidencia estadística que soporte la afirmación acerca de la superioridad del rendimiento de los distritos de riego respecto del de temporal. En consecuencias, no es válido argüir dicha superioridad de producción por hectárea de los distritos de riego para justificar el uso de las grandes cantidades de agua destinadas a tal fin. Además, se demuestra indirectamente la improcedencia de comparar dos procesos productivos totalmente diferentes, como se apuntó líneas arriba, y probablemente ponga de manifiesto que la tierra que no tenía riego - por ser tierra árida- al regarse aumenta su rendimiento. Esto es, comparado el rendimiento de la tierra árida consigo misma, pero no con la de temporal.

Se demostró que el rendimiento por hectárea de maíz grano es el mismo en temporal y en los distritos de riego. De esto se puede concluir que la tierra de temporal es más apta para el cultivo de maíz grano que la utilizada en los distritos de riego, y que el riego ha ido incorporando a las tierras marginales con un costo creciente, principalmente en términos de agua.

El resultado expuesto es relevante para la toma de decisiones y diseño de políticas públicas, toda vez que se ha tratado a la producción de maíz grano de temporal como inferior en rendimiento respecto del de los distritos de riego, cuando se ha demostrado que son iguales, por lo tanto, las políticas de apoyo a la producción por lo menos deben ser iguales para ambos, por lo menos, porque el temporal -o buen temporal, en el sentido de que no necesita agua para riego- no requiere de la construcción de grandes obras para almacenar y conducir el agua. En suma, la producción de maíz grano en temporal tiene un menor costo social y ambiental respecto de la obtenida en los distritos de riego, por lo menos en términos de agua. 


\section{Bibliografía}

Agroder, (2012), Producción 2010. Comparativo estatal, modalidad temporal y riego. < http://www.agroder.com/Documentos/Publicaciones/Produccion_de_Maiz_ en_Mexico-AgroDer_2012.pdf> 12 de noviembre de 2014, pp. 6.

Aguilar Soto, César, Sistemas de regadío y empresarios agrícolas en el norte de Sinaloa, México, 1900-1960. (Axe III, Symposium 14). Independencias-DependenciasInterdependencias, VI Congreso CeISAL 2010, Jun 2010, Toulouse, France. < https://halshs.archives-ouvertes.fr/halshs-00496951> 10 de julio de 2015, 2010, pp. 28.

Castillo-Caamal, J. B., Caamal-Maldonado, J. A. (2011),"Efecto de la fecha de siembra del fríjol terciopelo (Mucuna sp.) como cultivo de cobeertera en el rendimiento de maíz", Tropical and Subtropical Agroecosystems, enero-abril, pp. 101108.

Cerutti, M., La agriculturización del desierto. Estado, riego y agricultura en el norte de México (1925-1970), IV Encuentro. Asociación Española de Historia Económica, Monterrey, México, <http://www.econ.unavarra.es/ 4e_aehe/4EncuentroAEHE/ Programa_files/CeruttiMexico.pdf > el 02 de mayo de 2015, 2013,pp. 69.

Conagua (Comisión Nacional de Agua) (2014), Diagnóstico del programa presupuestario K141. Rehabilitación y modernización de infraestructura de riego y temporal tecnificado, Conagua, México, pp. 1-31 <http://www.coneval.gob.mx/Informes/Evaluacion/Diagnostico/Diagnostico_2014/Diagnostico_2014_SEMARNAT_K141. pdf> el 01 de junio de 2015.

-- (2014a), Estadísticas del agua en México, México, pp. 239.

-- (2014b), Estadísticas agrícolas de los Distritos de Riego. Año agrícola 2012-2013, México, pp. 379.

imta (Instituto Mexicano de Tecnología del Agua) (2015), < http://www.edistritos. com/DR/estadisticaAgricola/distrito.php> el 05 de marzo de 2015.

Kokler, Heinz (1999), Estadística para negocios y economía, CECSA, México, pp. 1053.

Ocampo Portillo, Minerva; Ortíz Solorio, Carlos; González Ríos, Jesús; González Mateos, Ricardo; Volke Haller, Víctor; Manzo Ramos, Fernando (2007), "Efecto de la erosión del suelo sobre el rendimiento de maíz de temporal", Terra Latinoamericana, vol. 25 num. Sin mes, pp. 399-408.

Palmer Viqueira, Jacinta y Tomás Martínez Saldaña, (s.f). Antropología del regadío. http://ceer.isa.utl.pt/cyted/mexico2006/tema\%201/6_JPalerm_Mexico.pdf 01 de mayo de 2015.

Pérez López, Delfina de Jesús; Rodríguez Pérez, Juan Enrique; Vázquez García, Luis Miguel; González Huerta, Andrés; Sahagún Castellanos, Jaime (2007). 
Economía Informa núm. 398 mayo - junio • 2016

Rendimiento del maíz de temporal y su relación con la pudrición de mazorca. Agricultura Técnica en México, enero-abril, 33-42.

Rivera González, Miguel, Julián Cerano Paredes, Ramón Trucíos Caciano, Julio Cesar Ríos Saucedo y Juan Estrada Ávalos (2012), “Actualización de la frontera agrícola en los distritos de riego a nivel nacional", Relación agua-suelo-planta-agrofaz,vol. 12, núm. 3, pp. 67-72.

Sagarpa (Secretaría de Agricultura, Ganadería, Desarrollo Rural, Pesca y Alimentación) (2008-2012), Programa de apoyo a la inversión en equipamiento e infraestructura. Memoria documental del "proyecto estratégico de tecnificación de riego 2008-2012", México, pp. 30.

SHCP (Secretaría de Hacienda y Crédito Público). Finanaciera Nacional de Desarrollo Agropecuario, Rural, Forestal y Pesquero (2014), "Panorama del Maíz". $<$ http://www.financierarural.gob.mx/informacionsectorrural/Panoramas/ Panorama\%20Ma\%C3\%ADz\%20\%28may\%202014\%29.pdf > el 08 de junio de 2015.

Soto Mora, C. (2003), "La agricultura comercial de los distritos de riego en México y su impacto en el desarrollo agrícola”. Investigaciones Geográficas, Boletín del Instituto de Geografía, unam. No. 50, México, pp. 173-195.

Stevenson, W. J (2002), Estadística para administración y economía, Oxford University Press, México, pp. 585.

Turrent Fernández, Antonio, Timothy A. Wise y Elise Garvey (2012), "Factibilidad de alcanzar el potencial productivo de maíz de México". Woodrow Wilson International Center for Scholars. También disponible en http://www.wilsoncenter.org/publication/mexican-rural-development-research-reports-englishspanish pp. 36.

Vargas-Velázquez, Sergio (2010), “Aspectos socioeconómicos de la agricultura de riego en la Cuenca Lerma-Chapala", Sociedad y Territorio, x, (32), El Colegio Mexiquense, A.C., pp. 231-263.

Varian, Hall (1992), Análisis microeconómico, Antoni Bosch, España, pp. 637.

Wackerly, D., William Mendenhall y Richard Scheaffer (2002), Estadística matemática con aplicaciones, Thomson, México, pp. 853. 